\title{
A parallel Numerical Algorithm for Solving Some Fractional Integral Equations
}

\author{
Khalid Mindeel Mohammed Al-Abrahemee \\ Khalid.mohammed@qu.edu.iq \\ Department of Mathematics, College of Education,University of AL-Qadisiyhah, Qadisiyhah,Iraq.
}

Article history: Received 12 August 2018, Accepted 26 September 2018, Publish January 2019

\begin{abstract}
In this study, He's parallel numerical algorithm by neural network is applied to type of integration of fractional equations is Abel's integral equations of the $1^{\text {st }}$ and $2^{\text {nd }}$ kinds. Using a Levenberge - Marquaradt training algorithm as a tool to train the network. To show the efficiency of the method, some type of Abel's integral equations is solved as numerical examples. Numerical results show that the new method is very efficient problems with high accuracy.
\end{abstract}

Keywords: Neural network, Abel's integral equations, Levenberg - Marquardt training algorithm.

\section{Introduction}

Abel's equations are interconnected to a wide-ranging of physecal problems, such as temperature transfer [1], nonlinear diffusion [2], the propagation of nonlinear waves [3], and were used in the theory of nautron transportation and traffic theory. There is several methods counting numerical analysis thus far away to reviewing this type of integration as well as their variations with several uses [4-5]. In [6] he also talked about solutions to this type of integration under assured conditions by some special ways.

Sumner calculated this type from the point of observation of the convolutional convert studied a numerical solution for this integration using orthogonal polynomials. Hilbert worked on the problem of nonlinear type of energy, explained in locked shaped by expressive a sectional holomorphic function by earnings of an integral with energy nucleus, and converted the problem to one of resolving a generalized reviewing this type of integration.

In [7] used the reverse of several of fractional order. In [8] resolved this integration numerically It is based on the process of approximation of partial integrations and derivatives. In [9] the researcher used Chebyshev polynomials for solve this integration. In [10] for easy calculations the researcher used some conversions such homotopy pertuarbation and Laplace transforms algorithm.

\section{Abel's Integral Equations}

This type of integration studied by Nieal Henreik Abel and Liouveille for its real importance in modeling many phenomena in mechanical, electronic, engineering, chemical and basic sciences [1113]. Where it emerged for this type of integration two types first and second as follows respectably 


$$
f(x)=\lambda \int_{0}^{x} \frac{y(t)}{(x-t)^{\alpha}} d t
$$

And

$$
y(x)=f(x)+\lambda \int_{0}^{x} \frac{y(t)}{(x-t)^{\alpha}} d t
$$

Where $\lambda, \alpha$ and $f(x)$ and the function are given where $0 \leq \alpha \leq 1, f(c) \in c[0,1], 0 \leq x, t \leq$ $T$ and $T$ is constant.

We will solve this type of integration of fractional equations by means of neural networks using three layers: the input and output layers and the hidden layer contain 7 hidden units and one linear output unit, the sigmoid activation function of each unit in hidden layer is tanseg. function by a type of training, the Levenberge - Marqaurdt training algoreithm is used to train the network.

\section{Description of the Method}

In this part we will describe how our method can be used to discovery the approximate result of Equations (1) and (2) let $\gamma(\chi)$ denotes the result to be calculated, $\left.\gamma_{\tau}\left(\chi_{i}, \rho\right)\right)$ refers to the analytical solution. In the proposed approach, the FFNN experimental solution is used and the factors $\rho$ correspond to the weights and bias of the neural architecture. We choose a model for a pilot function $\mathrm{yt}(\mathrm{x})$ to meet $\mathrm{BC}$ requirements. This is attained by lettering it as two sets:

$\gamma_{\tau}\left(\chi_{i}, \rho\right)=\phi(\chi)+\xi(\chi, \mathrm{N}(\chi, \rho))$

where $\mathrm{N}(\chi, \rho)$ is a singleoutput FFNN with $\rho$ factors and one input element breast-feeding on the $\chi$ input vector.

The part $\phi(\chi)$ does not contain parameters that can be adjusted and meet the boundary conditions. The second term $\xi(\chi, \mathrm{N}(\chi, \rho))$ is created so that it does not contribute to $\mathrm{BC}$, because

$\gamma_{\tau}(\chi)$ satisfies them. This part can be molded using FFNN whose weights and biases must be adjusted to address the minimization problem.

\section{Illustration of the Method}

To explain the method, Let the equations $((1)$ and (2)), where $\mathrm{x} \in[0,1]$ and the $\mathrm{BC}: \mathrm{y}(0)=\mathrm{A}$ and $y(1)=\beta$, the approximation of solution can be written as:

$\gamma_{\tau}\left(\chi_{i}, \rho\right)=\mathrm{A}+(\beta-\mathrm{A}) \chi+\chi(\chi-1) \mathrm{N}(\chi, \rho)$

The error calculated to be minimized is given by

$$
E[p]=\left\{\frac{d^{2} \gamma_{t}}{d \chi^{2}} \sum_{i=1}^{n} f\left(\gamma_{i}, \gamma_{t}^{\prime}, \chi_{i}\right)+\int_{0}^{\chi} \frac{\gamma(t)}{(\chi-t)^{\alpha}} d t\right\}^{2}
$$


where the xi's are points in $[a, b]$.

\section{Numerical Examples}

In this section we will apply the above algorithm to three different examples of the first kind and second kind. For each test problem the analytic solution $\gamma_{a}(\chi)$ was identified in improvement. So, we have tried the accuracy of the solutions obtained through the equation;

$$
\Delta y(x)=\left|\gamma_{t}(\chi)-\gamma_{a}(\chi)\right|
$$

\section{Example 1}

In the first example we will demonstrate the work of the proposed algorithm on the first type of fractional equations for that integration [14]

$$
\int_{0}^{x} \frac{\mathrm{U}(t)}{(x-t)^{\frac{1}{3}}} d t=x^{\frac{5}{3}}, \quad 0 \leq \times \leq 1, \text { where the exact solution } \cup(t)=\frac{10 \times}{9}
$$

\begin{tabular}{|c|c|c|c|}
\hline input & Analytic solution & $\begin{array}{l}\text { Out of suggested FFNN } y_{t}(x) \\
\text { for LM training algorithm }\end{array}$ & $\begin{array}{c}\text { The error } E(x)=\left|y_{t}(x)-y_{a}(x)\right| \\
\text { w h e r e } y_{t}(x) \text { computed by the } \\
\text { following training algorithm ( Trainlm) }\end{array}$ \\
\hline $\mathrm{x}$ & $\mathrm{y}_{\mathrm{a}}(\mathrm{x})$ & Trainlm & \\
\hline 0.0 & 0.00000000000000 & 0.00000000000000 & 0.00000000000000 \\
\hline 0.1 & 0.11111111111111 & 0.111111134005765 & 0.00000002289465 \\
\hline 0.2 & 0.22222222222222 & 0.22298600387254 & 0.00076378165032 \\
\hline 0.3 & 0.33333333333333 & 0.33333333398739 & 0.00000000065406 \\
\hline 0.4 & 0.44444444444444 & 0.44444456447638 & 0.00000012003194 \\
\hline 0.5 & 0.55555555555556 & 0.55555555347291 & 0.00000000208265 \\
\hline 0.6 & 0.666666666666667 & 0.66666666684937 & 0.00000000018270 \\
\hline 0.7 & 0.77777777777778 & 0.77777342990821 & 0.00000434786957 \\
\hline 0.8 & 0.88888888888889 & 0.88888888534289 & 0.00000000354600 \\
\hline 0.9 & 1.00000000000000 & 1 & 0.00000000000000 \\
\hline 1.0 & 1.11111111111111 & 1.12122256799995 & -0.01011145688884 \\
\hline
\end{tabular}

Table 1. Analytic and Neural solution of example 1 
Table 2. The performance of the train with epoch and time

\begin{tabular}{|c|c|c|c|c|}
\hline Train Function & Performance of train & Epoch & Time & MSE \\
\hline Trainlm & $2.39-31$ & 50 & $0: 00: 02$ & $9.34772 \mathrm{E}-06$ \\
\hline
\end{tabular}

Table 3. Initial weight and bias of the network for different training algorithm

\begin{tabular}{|c|c|c|c|}
\hline \multicolumn{4}{|c|}{ Weights and bias for trainlm } \\
\hline Net.IW $\{1,1\}$ & Net.IU $\{1,1\}$ & Net.LW $\{2,1\}$ & Net.B $\{1\}$ \\
\hline 0.2436 & 0.9876 & 0.1239 & 0.4876 \\
\hline 0.4563 & 0.9843 & 0.6398 & 0.2542 \\
\hline 0.6534 & 0.0987 & 0.2760 & 0.1984 \\
\hline 0.4237 & 0.2341 & 0.0834 & 0.1295 \\
\hline 0.3122 & 0.7523 & 0.2314 & 0.5934 \\
\hline
\end{tabular}

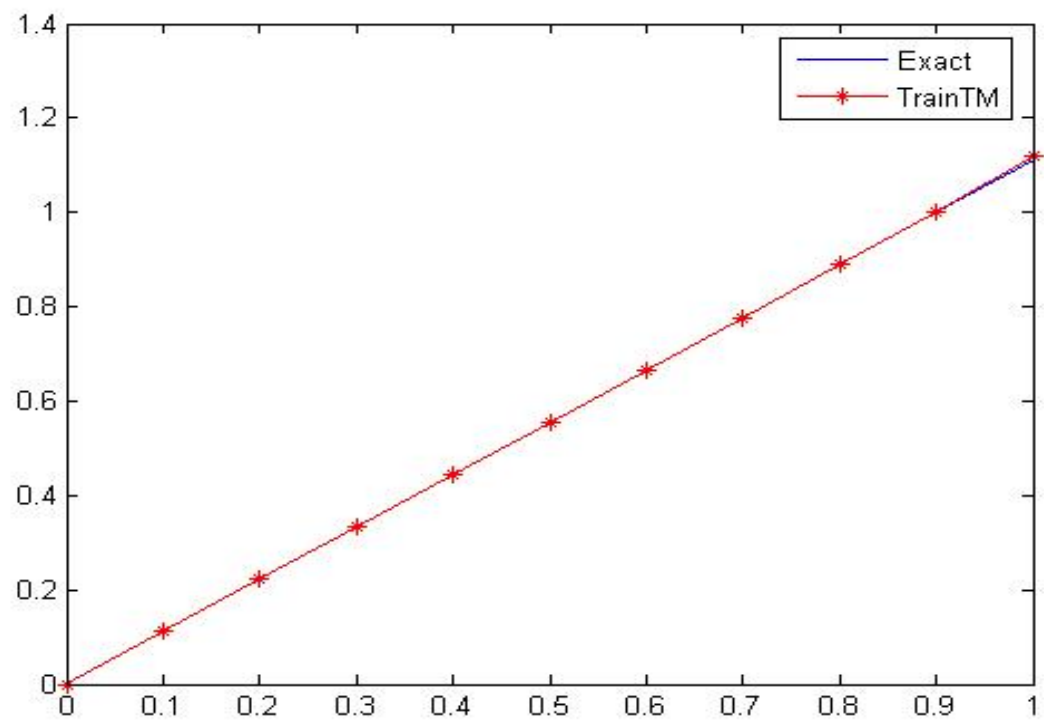

Figure 1. Exact and Approximate solution of example1 using: Modify Trainlm \& Trainlm Algorithms

\section{Example 2}

In this example we will demonstrate the work of the offered algorithm on the first type of fractional equations for that integration [15]

$$
\int_{0}^{x} \frac{\mathrm{U}(t)}{(\times-t)^{1 / 2}} d t=\frac{4}{105} \times^{\frac{3}{2}}\left(35-24 \times^{2}\right) 0 \leq \times \leq 1 \text {, where the exact solution is: } \cup(t)=\times-x^{3}
$$


Table 4. Analytic and Neural solution of example 2

\begin{tabular}{|c|c|c|c|}
\hline input & Analytic solution & $\begin{array}{c}\text { Out of suggested FFNN } \\
y_{t}(x) \text { for LM training } \\
\text { algorithm }\end{array}$ & $\begin{array}{c}\text { The error } E(x)=\left|y_{t}(x)-y_{a}(x)\right| \\
\text { wh e r e } y_{t}(x) \text { computed by the } \\
\text { following training algorithm( } \\
\text { Trainlm) }\end{array}$ \\
\hline $\mathrm{x}$ & $\mathrm{y}_{\mathrm{a}}(\mathrm{x})$ & Trainlm & \\
\hline 0.0 & 0.00000000000000 & 0 & 0.00000000000000 \\
\hline 0.1 & 0.09900000000000 & 0.09900465434298 & 0.00000465434298 \\
\hline 0.2 & 0.19200000000000 & 0.19204536657245 & 0.00004536657245 \\
\hline 0.3 & 0.27300000000000 & 0.27306587229787 & 0.00006587229787 \\
\hline 0.4 & 0.33600000000000 & 0.33500056477396 & 0.00099943522604 \\
\hline 0.5 & 0.37500000000000 & 0.37500056477342 & 0.00000056477342 \\
\hline 0.6 & 0.38400000000000 & 0.38400035428878 & 0.00000035428878 \\
\hline 0.7 & 0.35700000000000 & 0.35700675534198 & 0.00000675534198 \\
\hline 0.8 & 0.28800000000000 & 0.28774553609866 & 0.00025446390134 \\
\hline 0.9 & 0.17100000000000 & 0.17100645337243 & 0.00000645337243 \\
\hline 1.0 & 0.00000000000000 & 0 & 0.00000000000000 \\
\hline
\end{tabular}

Table 5. The performance of the train with epoch and time

\begin{tabular}{|c|c|c|c|c|}
\hline Train Function & Performance of train & Epoch & Time & MSE \\
\hline Trainlm & $2.38-31$ & 300 & $0: 00: 10$ & $9.72845 \mathrm{E}-08$ \\
\hline
\end{tabular}

Table 6. Initial weight and bias of the network for different training algorithm

\begin{tabular}{|c|c|c|c|}
\hline \multicolumn{4}{|c|}{ Weights and bias for trainlm } \\
\hline Net.IW $\{\mathbf{1 , 1}\}$ & Net.IU $\{\mathbf{1 , 1}\}$ & Net.LW $\{\mathbf{2 , 1}\}$ & Net.B $\{\mathbf{1}\}$ \\
\hline 0.6574 & 0.0975 & 0.9365 & 0.1233 \\
\hline 0.9786 & 0.4352 & 0.9284 & 0.7564 \\
\hline 0.4352 & 0.7564 & 0.5542 & 0.8735 \\
\hline 0.9807 & 0.9786 & 0.8635 & 0.9285 \\
\hline 0.5543 & 0.9843 & 0.6657 & 0.5637 \\
\hline
\end{tabular}




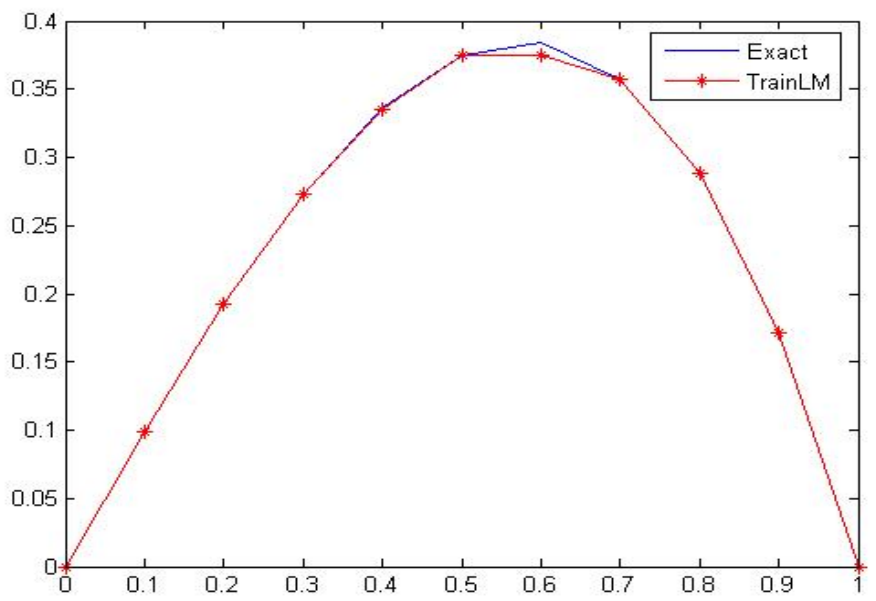

Figure 2. Exact \& Approximate solution of example1 by using: Modify Trainlm \& Trainlm Algorithms

\section{Example 3}

In the third example we will demonstrate the proposed technique on the second type of fractional equations for that integration [16]

$$
\boldsymbol{u}(\times)=\times^{2}+\frac{16}{15} \times^{\frac{5}{2}}-\int_{0}^{x} \frac{\cup(t)}{(\times-t)^{\frac{1}{2}}} d t \quad 0 \leq \times \leq 1, \text { where the exact solution is } \cup(t)=\times^{2}
$$

Table 7. Analytic and Neural solution of example 3

\begin{tabular}{|c|c|c|c|}
\hline input & Analytic solution & $\begin{array}{c}\text { Out of suggested FFNN } \\
y_{t}(x) \text { for LM training } \\
\text { algorithm }\end{array}$ & $\begin{array}{c}\text { The error } E(x)=\left|y_{t}(x)-y_{a}(x)\right| \\
\text { wh e r e } y_{t}(x) \text { computed by the } \\
\text { following training algorithm } \\
\text { (Trainlm) }\end{array}$ \\
\hline $\mathrm{x}$ & $\mathrm{y}_{\mathrm{a}}(\mathrm{x})$ & Trainlm & \\
\hline 0.0 & 0.00000000000000 & 0.00000000000000 & 0.00000000000000 \\
\hline 0.1 & 0.01000000000000 & 0.01000435620866 & 0.00000435620866 \\
\hline 0.2 & 0.04000000000000 & 0.04000065323146 & 0.00000065323146 \\
\hline 0.3 & 0.09000000000000 & 0.09000053427465 & 0.00000053427465 \\
\hline 0.4 & 0.16000000000000 & 0.16000063527763 & 0.00000063527763 \\
\hline 0.5 & 0.25000000000000 & 0.25000000624242 & 0.00000000624242 \\
\hline 0.6 & 0.36000000000000 & 0.36000342553427 & 0.00000342553427 \\
\hline 0.7 & 0.49000000000000 & 0.4903427761345 & 0.00034277613450 \\
\hline 0.8 & 0.64000000000000 & 0.6414366287593 & 0.00143662875930 \\
\hline 0.9 & 0.81000000000000 & 0.81034287614338 & 0.00034287614338 \\
\hline 1.0 & 1.00000000000000 & 1 & 0.00000000000000 \\
\hline
\end{tabular}


Table 8. The performance of the train with epoch and time

\begin{tabular}{|c|c|c|c|c|}
\hline Train Function & Performance of train & Epoch & Time & MSE \\
\hline Trainlm & $3.38-31$ & 700 & $0: 00: 11$ & $2.08999 \mathrm{E}-07$ \\
\hline
\end{tabular}

Table 9. Initial weight and bias of the network for different training algorithm

\begin{tabular}{|c|c|c|c|}
\hline \multicolumn{4}{|c|}{ Weights and bias for trainlm } \\
\hline Net.IW $\{\mathbf{1 , 1}\}$ & Net.IU $\{\mathbf{1 , 1}\}$ & Net.LW $\{\mathbf{2 , 1}\}$ & Net.B $\{\mathbf{1}\}$ \\
\hline 0.7645 & 0.6477 & 0.9573 & 0.9275 \\
\hline 0.8735 & 0.6298 & 0.3624 & 0.9539 \\
\hline 0.1249 & 0.6429 & 0.8729 & 0.2365 \\
\hline 0.9823 & 0.2314 & 0.7563 & 0.1126 \\
\hline 0.8669 & 0.3425 & 0.8365 & 0.7403 \\
\hline
\end{tabular}

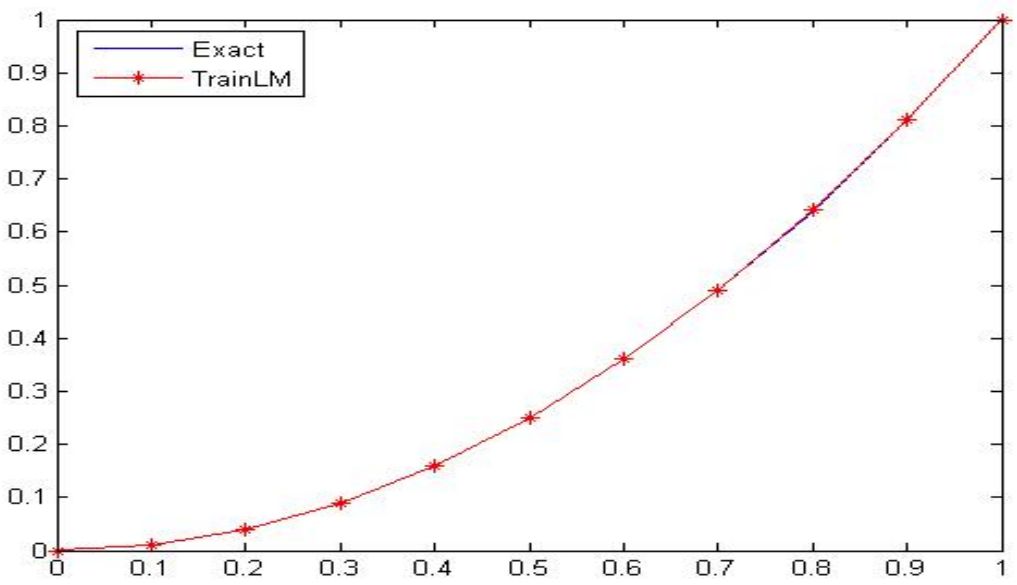

Figure 3. Exact \& Approximate solution of example1 using: Modify Trainlm \& Trainlm Algorithms

\section{Conclusions}

In this paper, a new procedure was used to solve a special type of fractional integrals using parallel processors and a high-level training algorithm. Then applied in three examples of different types were the results compared with analytical solutions for these integrals. The results show the accuracy of suggested method is very high and the speed of convergence is ideal. 


\section{References}

1. Mann,W.R.; Wolf, F. Heat transfer between solids and gases under nonlinear boundary conditions. Appl Math. 1951, 9, 163-184.

2. Goncerzewicz, J.; Marcinkowska, H.; Okrasinski,W.; Tabisz, K. On percolation of water from a cylindrical reservoir into the surrounding soil. Appl. Math. 1951, 16, 249-261.

3. Keller, J.J. Propagation of simple nonlinear waves in gas filled tubes with friction. Z. Angew. Math. Phys. 1981, 32, 170-181.

4. Lima, P.; Diogo, T. Numerical solution of nonuniquely solvable volterra integral equation using extrapolation methods. J. Comput. App. Math. 2002, 140, 537-557.

5. Singh, O.P.; Singh, V.K.; Pandey, R.K. A stable numerical inversion of Abel's integral equation using almost Bernstein operational matrix. J. Quant. Spectrosc. Radiat. Transf. 2010, 111, 245252.

6. Bushell, P.J.; Okrasinski,W. Nonlinear Volterra integral equations with convolution kernel. J. Lond. Math. Soc. 1990, 41, 503-510.

7. Li, M.; Zhao, W. Solving Abel's type integral equation with Mikusinski's operator of fractional order, Adv. Math. Phys. 2013. doi:10.1155/2013/806984.

8. Jahanshahi, S.; Babolian, E.; Torres, D.; Vahidi, A. Solving Abel integral equations of first kind via fractional calculus. J. King Saud Univ. Sci. 2015, 27, 161-167.

9. Saleh, M.H.; Amer, S.M.; Mohamed, D.S.; Mahdy, A.E. Fractional calculus for solving generalized Abel's integral equations suing Chebyshev polynomials. Int. J. Comput. Appl. 2014, 100, 19-23.

10. Kumar, S.; Kumar, A.; Kumar, D.; Singh, J.; Singh, A. Analytical solution of Abel integral equation arising in astrophysics via Laplace transform. J. Egypt. Math. Soc. 2015, 23, 102-107.

11. Wazwaz, A. M. Partial differential equations and solitary waves theory. Springer. Beijing and Berlin. 2009. ISBN 978-3-642-00250-2, e-ISBN 978-3-642-00251-9.

12. Rashidinia, J.; Tahmasebi, A. Approximate solution of linear integro differential equations by using modified Taylor expansion method. World journal of Modelling and Simulation. 2013, 9, 4, 289-301.

13. Abdulkawi, M.; Eshkuvatov, Z. K.; Nik Long, N. M. A. A note on the numerical solution of singular integral equations of cauchy type. world academy of science, engineering and technology. 2009, 3, 9, 893-896.

14. Huang, L.; Huang, Y.; Li, X-F. Approximate solution of Abel integral equation. Comput. Math. Appl. 2008, 56, 1748-1757.

15. Liu, Y-P.; Tao, L. High accuracy combination algorithm and a posteriori error estimation for solving the first kind Abel integral equations. Appl. Math. Comput. 2006, 178, 441- 451.

16. Kumar, S.; Kumar, A.; Kumar, D.; Singh J.; Singh, A. Analytical solution of Abel integral equation arising in astrophysics via Laplace transform. Journal Egypt. Math. Soc. 2014, 2, doi: org/10.1016/j.joems.2014.02.004.

17. Al-Abrahemee, K.M.M. Solution of Some Application of System of Ordinary Initial Value Problems Using Osculatory Interpolation Technique. Ibn Al-Haitham Journal for Pure and Applied Science. 2012, 25, 1. 
18. Tawfiq, L.N.M. On Training of Artificial Neural Networks. Al-Fatih journal. 2005,1,23,130-139.

19. Tawfiq, L. N. M; Yassien. S. M. Solution of High Order Ordinary Boundary Value Problems Using Semi-Analytic Technique. Ibn Al-Haitham Jour. for Pure \& Appl. Sci. 2013, 26, 1.

20. Tawfiq, L. N. M.; Hussein, R. W. On Solution of Regular Singular Initial Value Problems. Ibn Al-Haitham Jour. for Pure \& Appl. Sci. 2013, 26, 1.

21. Tawfiq, L. N. M.; Rasheed, H. W. On Solution of Regular Singular Ordinary Boundary Value Problem. Ibn Al-Haitham Jour. for Pure \& Appl. Sci. 2013, 26, 2. 